Relations industrielles

Industrial Relations

\title{
Index alphabétique des articles
}

Volume 26, numéro 4, 1971

URI : https://id.erudit.org/iderudit/028287ar

DOI : https://doi.org/10.7202/028287ar

Aller au sommaire du numéro

Éditeur(s)

Département des relations industrielles de l'Université Laval

ISSN

0034-379X (imprimé)

1703-8138 (numérique)

Découvrir la revue

Citer ce document

(1971). Index alphabétique des articles. Relations industrielles / Industrial

Relations, 26(4), 1074-1077. https://doi.org/10.7202/028287ar

Tous droits réservés (C) Département des relations industrielles de l'Université Laval, 1971
Ce document est protégé par la loi sur le droit d'auteur. L’utilisation des services d'Érudit (y compris la reproduction) est assujettie à sa politique d'utilisation que vous pouvez consulter en ligne.

https://apropos.erudit.org/fr/usagers/politique-dutilisation/ 


\section{INDEX - VOL. 26 - 1971}

\section{Index alphabétiques des articles}

Action (l') politique du CTC et de la FAT-COI : une comparaison depuis 1956, Jean Boivin, No 3

American Transfer Policies: Costs and Benefits, William F. Glueck, No 3

Analyse (une) de la nouvelle loi britannique sur les relations industrielles,

T. G. Whittingham et B. Towers, No 3

Aspect (l') économique de l'adaptation au changement technologique, Arthur A. Kruger, No 2

Bilan (le) de l'année 1970 en droit du travail français, G. H. Camerlynck, No 2

British Collective Bargaining: The Challenges of the 1970's, Robert F. Banks, No 3

British (the) Industrial Relations Bill : An Analysis, T.G. Whittingham et B. Towers, No 3

$620-639$

Caractère (le) majoritaire des principes d'accréditation, Pierre Verge, No 3 764-768

Centralized Collective Bargaining: U.S.-Canada Experience, Bryan M. Downie, No 1

Changements technologiques et techniques de licenciements, David Phillips Ross, No 4

Chronologie de la Législation Canadienne en matière de Relations du Travail (1968-1971), No 4

Collective agreements and their seniority provisions, Felix Quinet, No 4 900-906

Collective bargaining and Wage Equalization in Canada's Iron \& Steel Industry, (1939-1964), Bryan C. Williams, No 2

Compulsory Arbitration in British Columbia, Bill 33, Peter Z.W. Tsong, No 3

Conseil (le) consultatif du Travail et de la main-d'oeuvre publie son rapport annuel (deuxième) pour l'année 1970-1971, No 3

Conventions (les) collectives et leurs clauses d'ancienneté, Felix Quinet, No 4

$890-900$

Countercyclical (A) Training Program for Canada, Keith Newton, No 4 $865-888$

Coûts et Bénéfices des politiques américaines de mutation du personnel de cadre, William F. Glueck, No 3

Decentralized Bargaining: Its Problems and Direction in Ontario and the Western Provinces, J. Douglas Muir, No 1

Défis (les) posés à la négociation collective en Angleterre, Robert F. Banks, No 3

Economic (an) critique of current Technological change displacement techniques, David Phillips Ross, No 4 
Eléments d'une stratégie de l'entreprise. (Centre des dirigeants d'entreprise), No 4

Elements of a strategy for business. (Centre des dirigeants d'entreprise), No 4

Embauchage (l') d'un salarié lié par une convention collective, Pierre Verge, No 2

Entreprises (les) multi-nationales et les relations du travail: l'approche européenne, Paul Malles, No 1

Evaluation d'un programme de formation professionnelle, Jean Sexton, No 3

Evaluation of a training Program Set-up by a Relocation Committee, Jean Sexton, No 3

Extension and Feasability of the Woods Report to the Developing Countries, Syed M. A. Hameed, No 3

Human Adjustment to technological change: An Economist View, Arthur A. Kruger, No 2

Impact (l') économique des avantages sociaux, Robert Swidinsky, No 4

922-923

Industry-Wide Bargaining: A panacea?, Bernard Brody, No 2

Industry-Wide Bargaining by legislation: the Quebec Construction Industry, Gérard Hébert, No 1

$120-123$

Ingénieurs (les) sur le marché du travail en Amérique du Nord, Archibald D. Boyd, Andrew C. Gross, Raymond R. McKay, No 4

849-851

Inventaire (l') des emplois disponibles comme outil d'information sur le marché du travail dans les pays en voie de développement, Ozay Mehmet, No 3

706-707

Investment-Wages: theory and application, Gerhard Reber, No 2

Job Vacancy Survey as a tool of Labour Market information in developing countries, Ozay Mehmet, No 3

692-706

Judicial Review of Labour Arbitration in Ontario, Mark Thompson, No 2

471-489

Labor (the) market experience of Engineers in North America, Archibald D. Boyd, Andrew C. Gross, Raymond R. McKay, No 4

Labor Mobility: An investment in human Capital Approach, Athenas P. Kottis, No 4

Labor Relations in Ontario Hospitals: A question of Survival, Fraser Isbester and Sandra Castle, No 2

Lighting Problems in the enterprise, Pierrette Sartin, No 4

Livre (le) blanc sur l'assurance-chômage: une évaluation, Yvon Valcin, No 2

Meilleures relations du travail dans les hôpitaux ontariens: Condition de survie?, Fraser Isbester et Sandra Castle, No 2

Mobilité (la) du travail vue sous la forme d'un investissement, Athenas P. Kottis, No 4

Multi-Employer Bargaining: The Lumber Industry in British Columbia, 
Multi-Employer Bargaining: The Trucking Industry in Ontario, Graeme McKechnie, No 1

Multinational (the) Corporation and Industrial Relations: The European Approach, Paul Malles, No 1

Négociation (la) collective dans l'industrie canadienne du fer et de l'acier: 1939-1964, Bryan C. Williams, No 2

Négociation (la) collective sectorielle, Robert Sauvé, No 1

Négociation (la) multi-employeur: l'exploitation forestière en ColombieBritannique, Stuart Jamieson, No 1

$167-168$

Négociation (la) multi-employeur: l'industrie du camionnage dans l'Ontario, Graeme McKechnie, No 1

Négociation (la) sectorielle par décision de l'Etat : le cas de la construction au Québec, Gérard Hébert, No 1

Négociation (la) sectorielle : une panacée?, Bernard Brody, No 2

Négociations (les) collectives Canado-Américaines, Bryan M. Downie, No

Organized Labor Political Action: A comparison of the CLC and AFLCIO Experiences since 1956, Jean Boivin, No 3

$571-574$

Problèmes et tendances de la négociation collective décentralisée dans les systèmes d'éducation publique de l'Ontario et des provinces de l'Ouest, J. Douglas Muir, No 1

$144-145$

Problèmes posés dans l'entreprise par l'éclairage des locaux, Pierrette Sartin, No 4

951-969

Programme de formation anticyclique au Canada, Keith Newton, No 4

Rapport (le) Woods et les pays en voie de développement, Syed M. A. Hameed, No 3

888-889

$590-591$

Réforme (la) de la législation du travail en Ontario, 1970, John Crispo, No 4

864

Reform of the Status Quo Ontario's Bill 167, John Crispo, No 4

Regional Unemployment Rates and total Economic Growth in Canada, 1953-1968, Arthur W. Donner, No 3

$721-724$

Reopeners: The Task force on labour relations, and Freedman, David Phillips Ross, No 1

213-222

Role (the) and Functions of Industrial Relations Centres in France, Pierre Candau, No 3

$742-743$

Rôle (le) de la recherche dans la négociation collective centralisée, Felix Quinet, No 1

184-201

Rôle (le) de l'enquête dans le Code du Travail, Claude D'Aoust, No 1 229-233

Rôle (le) et les fonctions des centres de relations industrielles en France, Pierre Candau, No 3

726-742

Role (the) of Research in Centralized Bargaining: The Pay Research Bureau, Felix Quinet, No 1

Salaire (le) d'investissement : principes et mise en pratique, Gerhard Reber 394-398

Sectorial Collective Bargaining, Robert Sauvé, No 1 
Some Comments on the Task force on labour relations and Freedman: a reply to David P. Ross, H.D. Woods, No 1

Syndicalisme (le) à l'heure du pluralisme et des changements de structure, Solomon Barkin, No 4

Syndicalisme (le) est-il la réponse au problème des cadres?, Roger Chartier, No 2

Taux (les) de chômage régionaux et la croissance économique générale au Canada: 1953-1968, Arthur W. Donner, No 3

Trade-Unions in an Age of Pluralism and structural change, Solomon Barkin, No 4

Unionization (is) the answer to management and supervision problems, Roger Chartier, No 2

Unité (l') d'accréditation appropriée et la langue du travail: l'affaire de la régionale Le Royer, Claude D'Aoust, No 2

Unité (l') d'accréditation des professionnels, Claude D'Aoust, No 3

768-772

Violation (la) de la convention collective peut-elle donner ouverture à des dommages-intérêts, Pierre Verge, No 1

White Paper (the) on unemployment insurance: An evaluation, Yvon Valcin, No 2

\section{Index méthodiques des articles}

\section{Généralités}

Problèmes posés dans l'entreprise par l'éclairage des locaux. (Pierrette Sartin). no 4 , p. 951

\section{Jurisprudence du travail}

L'embauchage d'un salarié lié par une convention collective peut-il être assorti de conditions particulières non contenues dans celle-ci et dans quelle mesure l'arbitre des griefs peut-il tenir compte d'une telle entente particulière. (Pierre Verge). no 2, p. 502

L'unité d'accréditation des professionnels. L'Article 20 du Code du Travail et la syndicalisation des ingénieurs et autres scientifiques. Jugement du tribunal du travail dans le cas de la RCA Limited. (Claude D'Aoust). no 3, p. 768 L'unité de négociation appropriée et la langue de travail: l'affaire de la régionale Le Royer. (Claude D'Aoust). no 2, p. 497

Le caractère majoritaire, principe d'accréditation. La Cour Suprême du Canada réaffirme le droit du syndicat majoritaire à l'accréditation. (Pierre Verge). no 3, p. 764

\section{Législation du travail - Canada}

Chronologie de la Législation Canadienne en matière de relations du travail, (1868-1971). no 4 , p. 1013

Le caractère majoritaire, principe d'accréditation. La Cour Suprême du Canada réaffirme le droit du syndicat majoritaire à l'accréditation. (Pierre Verge). 